\title{
Discontinuous Extraction of a Nonrenewable Resource
}

\author{
Eric Iksoon $\operatorname{Im}^{1}$ \\ Professor of Economics \\ Department of Economics, University of Hawaii at Hilo, Hilo, Hawaii \\ Ujjayant Chakravorty \\ Professor of Economics \\ Department of Economics, University of Central Florida, Orlando, Florida \\ James Roumasset \\ Professor of Economics \\ Department of Economics, University of Hawaii at Manoa, Honolulu, Hawaii
}

\begin{abstract}
This paper examines the sequence of optimal extraction of nonrenewable resources in the presence of multiple demands. We provide conditions under which extraction of a nonrenewable resource may be discontinuous over the course of its depletion.
\end{abstract}

JEL classification: Q3; Q4

Keywords: Backstop technology; Dynamic optimization; Energy resources; Herfindahl principle; Multiple demands

${ }^{1}$ Eric Iksoon Im, 200 W. Kawili Steet, Hilo, Hawaii 96720-4091; ph: (808)974-7467; fax: (808)974-7685; email: eim@hawaii.edu. We would like to thank an anonymous referee for valuable comments. 


\section{Introduction}

A fundamental theorem of resource economics is that when there is a single end-use, the optimal sequence for extracting deposits of a nonrenewable resource should be in the order of their effective unit-costs of extraction (e.g., Herfindahl (1967), Solow and Wan (1976), Lewis (1982). ${ }^{2}$ This principle has been generalized to the case of multiple enduses. To wit, resources will be extracted in order of their end-use specific prices, defined as the sum of extraction costs, conversion costs, transportation costs, and in situ shadow prices (Chakravorty and Krulce, 1994, henceforth CK; and Gaudet, Moreaux and Salant, 2001, GMS). ${ }^{3}$ By adding set-up costs to the basic model, GMS prove a "vacillation" result: one resource or landfill site may be temporarily abandoned in favor of a higher cost resource/site and utilized again at a later date. In this paper, we show that discontinuous extraction of a nonrenewable resource is possible, even without setup costs, and provide conditions under which the discontinuity occurs.

We modify the framework of CK who consider two nonrenewable resources, oil $(O)$ and coal $(C)$ and two end-uses, electricity $(E)$ and transportation $(T)$, each characterized by their own demand. We add a third backstop resource $(B)$ with an infinite supply (e.g., solar power). ${ }^{4}$ While the assumption of a constant unit extraction cost in CK is retained for each resource $\left(c_{i}, i=O, C, B\right)$, we specify conversion costs as both resource and

\footnotetext{
${ }^{2}$ For exceptions, see Kemp and Long (1980) and Amigues et al. (1998).

${ }^{3}$ The Kemp/Long and Amigues exceptions to the Herfindahl principle are consistent with this more general principle.

${ }^{4}$ GMS show that at least three resources are needed for discontinuous extraction.
} 
demand-specific $\left(z_{i j}, i=O, C, B ; j=E, T\right.$ ) so that the net cost of resource $i$ in demand $j$ is $w_{i j}=c_{i}+z_{i j}$.

The planner chooses instantaneous extraction rates of resource $i$ in demand $j$, denoted $q_{i j}(t)$, in order to maximize the discounted social surplus, $W$ :

$$
W=\int_{0}^{\infty} e^{-r t}\left[\sum_{j}\left(\int_{0}^{\sum_{i} q_{i j}} D_{j}^{-1}(x) d x\right)-\sum_{i, j}\left(c_{i}+z_{i j}\right) q_{i j}(t)-\sum_{i} \lambda_{i}(t) \sum_{j} q_{i j}(t)\right] d t
$$

subject to

$$
q_{i j}(t) \geq 0 ; \quad Q_{i}(t) \geq 0 ; \quad \dot{Q}_{i}(t)=-\sum_{j} q_{i j}(t)
$$

where $r$ denotes the discount rate, $D_{j}^{-1}$ the inverse demand function for $j, Q_{i}(t)$ the stock of resource $i$ available at time $t$ and $\lambda_{i}(t)$ the co-state variable for resource $i$. Define the equilibrium price for demand $j$ as $p_{j}(t)=D_{j}^{-1}\left(\sum_{i} q_{i j}(t)\right)$ and the price of resource $i$ in demand $j$ as $p_{i j}(t)=c_{i}+z_{i j}+\lambda_{i}(t) \equiv w_{i j}+\lambda_{i}(t)$. The necessary and sufficient conditions 5 are

$p_{j}(t) \leq p_{i j}(t) \quad\left(\right.$ if $<$ then $\left.q_{i j}(t)=0\right)$;

$\dot{\lambda}_{i}(t)=r \lambda_{i}(t)$

$\lim _{t \rightarrow \infty} e^{-r t} \lambda_{i}(t) \geq 0 ; \quad \lim _{t \rightarrow \infty} e^{-r t} \lambda_{i}(t) Q_{i}(t)=0$

\footnotetext{
${ }^{5}$ The proof of sufficiency is essentially the same as in CK, hence suppressed.
} 
Conditions (3) and (4) imply that $\lim _{t \rightarrow \infty} Q_{i}(t)=0$ for nonrenewable resource $i, i=O, C$, and $\lambda_{B}(0)=\lambda_{B}(t)=0$ for the backstop resource which is in infinite supply.

\section{Optimal Extraction Sequence}

Consider the case in which oil is the cheapest resource for both demands and the backstop is the most expensive. That is,

Assumption: $0<w_{O j}<w_{C j}<w_{B j}<\infty, \quad j=E, T$.

As shown by Chakravorty, Krulce, and Roumasset (2005), in general the ordering of the shadow prices is exactly the reverse of that of net costs, hence $0=\lambda_{B}(t)<\lambda_{C}(t)<\lambda_{O}(t)<\infty \quad \forall t \in[0, \infty)$. Since oil and coal are nonrenewable resources, they will be eventually exhausted and the backstop is used for both demands. By virtue of their Proposition 7, the extraction sequence in each demand follows the order of the net costs, i.e., oil followed by coal and then by the backstop resource (see Fig.1). Not every resource needs to be extracted for each demand.

\section{Conditions for Discontinuous Resource Extraction}

In this section, we demonstrate graphically the possibility of discontinuous extraction of a nonrenewable resource, and then provide necessary and sufficient conditions for the discontinuity to occur. In Fig.1, the (energy) resource price for each demand is depicted as an envelope curve: in bold solid for transportation and in bold dash for electricity. Coal is extracted in phase II and again in phase IV, but not in the intermediate phase III. 
$\longrightarrow p_{T}(t):$ Energy price for transportation

$p_{E}(t):$ Energy price for electricity

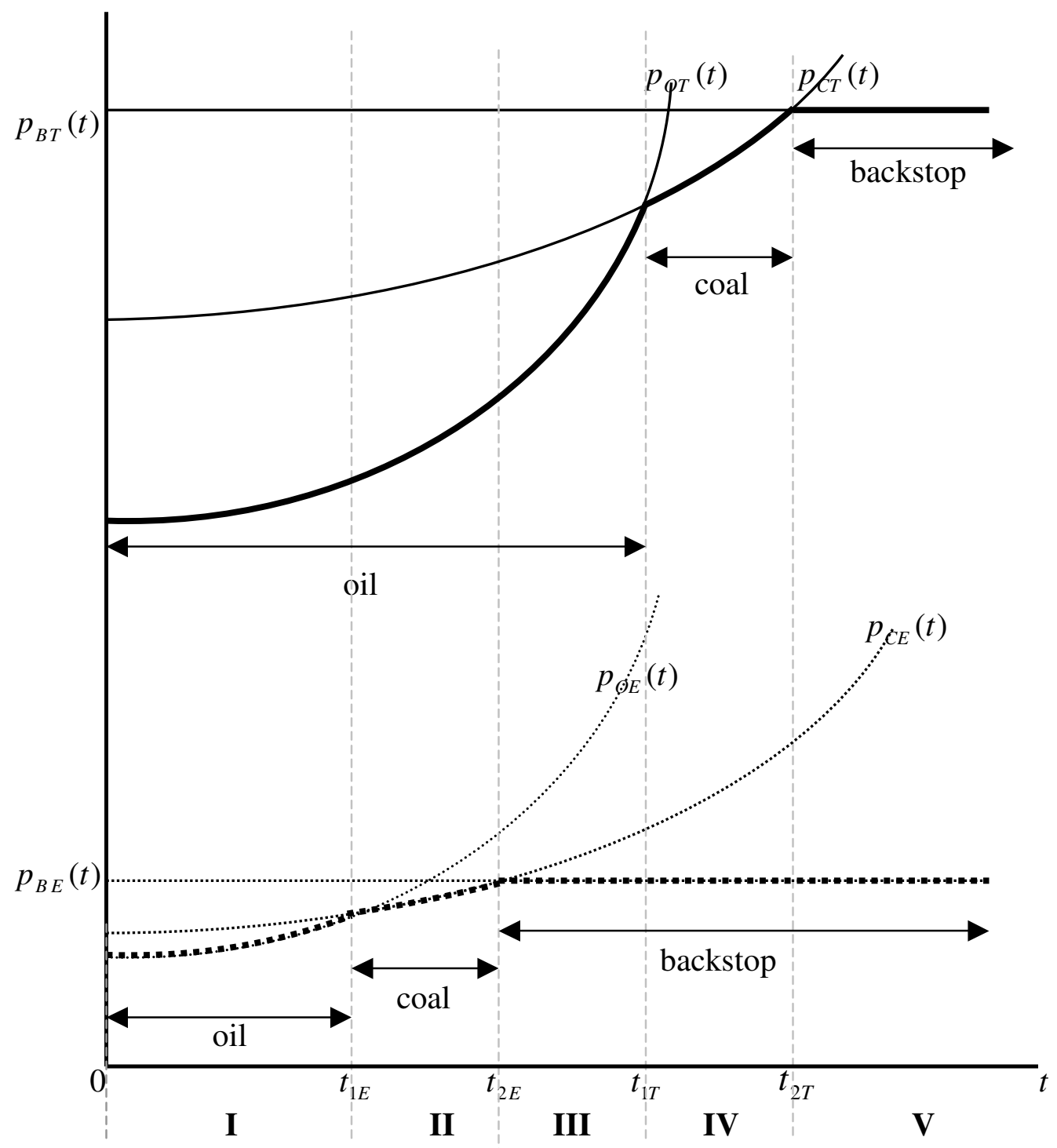

Fig. 1: Discontinuous Coal Extraction: Coal is extracted in phase II and IV, but not in III. 
The switch point sequence in Fig. 1 is S1: $0<t_{1 E}<t_{2 E}<t_{1 T}<t_{2 T}<\infty$ where $t_{1 j}$ and $t_{2 j}$ denote, respectively, oil-to-coal and coal-to-backstop switch points. If the $p_{O E}(t)$ curve in the lower part of Fig. 1 shifts up, oil may not be used in electricity, but coal extraction will remain discontinuous with an altered switch point sequence $S 2$ : $t_{1 E} \leq 0<t_{2 E}<t_{1 T}<t_{2 T}<\infty$. Either $S 1$ or $S 2$ is equivalent to the following three inequalities:

(i). $0<t_{2 E}$; (ii). $t_{2 E}<t_{1 T}$; (iii). $t_{1 j}<t_{2 j}, \quad j=E, T$.

Under these two sequences, coal is extracted first for electricity and then for transportation. We can now state

PROPOSITION: Given the Assumption in (5), coal is extracted discontinuously, first for electricity $(E)$ and then for transportation $(T)$ after a time delay, iff

(I). $\lambda_{C}(0)<w_{B E}-w_{C E}$ 
(II). $1+\max _{j}\left\{\frac{w_{C j}-w_{O j}}{w_{B j}-w_{C j}}\right\}<\frac{\lambda_{O}(0)}{\lambda_{C}(0)}<1+\frac{w_{C T}-w_{O T}}{w_{B E}-w_{C E}}, \quad j=E, T .^{6}$

Proof: At the switch points for demand $j, p_{O j}=p_{C j}$ and $p_{C j}=p_{B j}$ which yield:

$t_{1 j}=\frac{1}{r} \ln \frac{w_{C j}-w_{O j}}{\lambda_{O}(0)-\lambda_{C}(0)} ; \quad t_{2 j}=\frac{1}{r} \ln \frac{w_{B j}-w_{C j}}{\lambda_{C}(0)}$.

Substituting (7) into (6), noting $0<\lambda_{C}(0)<\lambda_{O}(0)<\infty$ from Section 2, we can rewrite (i),

(ii) and (iii) in terms of $\lambda_{i}(0)$ and $w_{i j}$ :

(i). $0<t_{2 E} \quad \Leftrightarrow \quad \lambda_{C}(0)<w_{B E}-w_{C E}$

(ii). $t_{2 E}<t_{1 T} \Leftrightarrow \frac{\lambda_{O}(0)}{\lambda_{C}(0)}<1+\frac{w_{C T}-w_{O T}}{w_{B E}-w_{C E}}$

(iii). $t_{1 j}<t_{2 j} \Leftrightarrow \frac{w_{C j}-w_{O j}}{\lambda_{O}(0)-\lambda_{C}(0)}<\frac{w_{B j}-w_{C j}}{\lambda_{C}(0)} \Leftrightarrow$

$$
\frac{\lambda_{O}(0)}{\lambda_{C}(0)}>1+\max _{j}\left\{\frac{w_{C j}-w_{O j}}{w_{B j}-w_{C j}}\right\}
$$

${ }^{6}$ There exists a subset of $w=\left(w_{O E}, w_{C E}, w_{B E}, w_{O T}, w_{C T}, w_{B T}\right)$ which admits conditions (I) and (II), e.g., $w=(4,5,6,1,4,6)$. Given $w, \lambda_{O}(0)$ and $\lambda_{C}(0)$ still depend on other factors such as the initial stocks of resources, the discount rate and the magnitude of demands, hence are not determined solely by $w$. 
Noting that (9) and (10) jointly are equivalent to (II) in the Proposition completes the proof.

The conditions in the Proposition can be re-expressed as three simple inequality constraints: $\lambda_{C}(0)<\alpha, \lambda_{O}(0)<\beta \lambda_{C}(0)$ and $\lambda_{O}(0)>\gamma \lambda_{C}(0)$ where

$$
\begin{aligned}
& \alpha \equiv w_{B E}-w_{C E}>0 ; \\
& \beta \equiv 1+\frac{w_{C T}-w_{O T}}{w_{B E}-w_{C E}}>1 ; \\
& \gamma \equiv 1+\max _{j}\left\{\frac{w_{C j}-w_{O j}}{w_{B j}-w_{C j}}\right\}>1,
\end{aligned}
$$

which are graphically depicted in Fig. 2. Let $\mathbb{F}=\left\{\left(\lambda_{C}(0), \lambda_{O}(0)\right) \mid 0<\lambda_{C}(0)<\lambda_{O}(0)<\infty\right\}$ which lies above the $45^{\circ}$ line in the figure. Set $\mathbb{F}$ defines the domain for $\left(\lambda_{C}(0), \lambda_{O}(0)\right)$ on which the discontinuous extraction of coal is feasible. The entire shaded area $(\mathbb{S})$ represents an open subset that satisfies the conditions for either $S 1$ or $S 2$ to occur. Note that $t_{1 E}>0$ for $S 1$ and $t_{1 E} \leq 0$ for $S 2$. Using $t_{1 j}$ in (7) for $j=E$, we can restate these two inequalities as $\lambda_{O}(0)>\mu+\lambda_{C}(0)$ for $S 1$ and $\lambda_{O}(0) \leq \mu+\lambda_{C}(0)$ for $S 2$, respectively, where $\mu \equiv w_{C E}-w_{O E}>0$. The line $\lambda_{O}(0)=\mu+\lambda_{C}(0)$ splits the entire shaded area in the figure into two parts. The dark shaded area $\left(\mathbb{S}_{1}\right)$ is an open subset of $\mathbb{S}$ that admits only sequence $S 1$ and the light shaded area $\left(\mathbb{S}-\mathbb{S}_{1}\right)$ admits only $S 2$. If $\mu \geq \mu^{*} \equiv \alpha(\beta-1)$, there 
exists no $\left(\lambda_{C}(0), \lambda_{O}(0)\right) \in \mathbb{S}$ which admits $S 1$, so that the entire shaded area admits only S2.

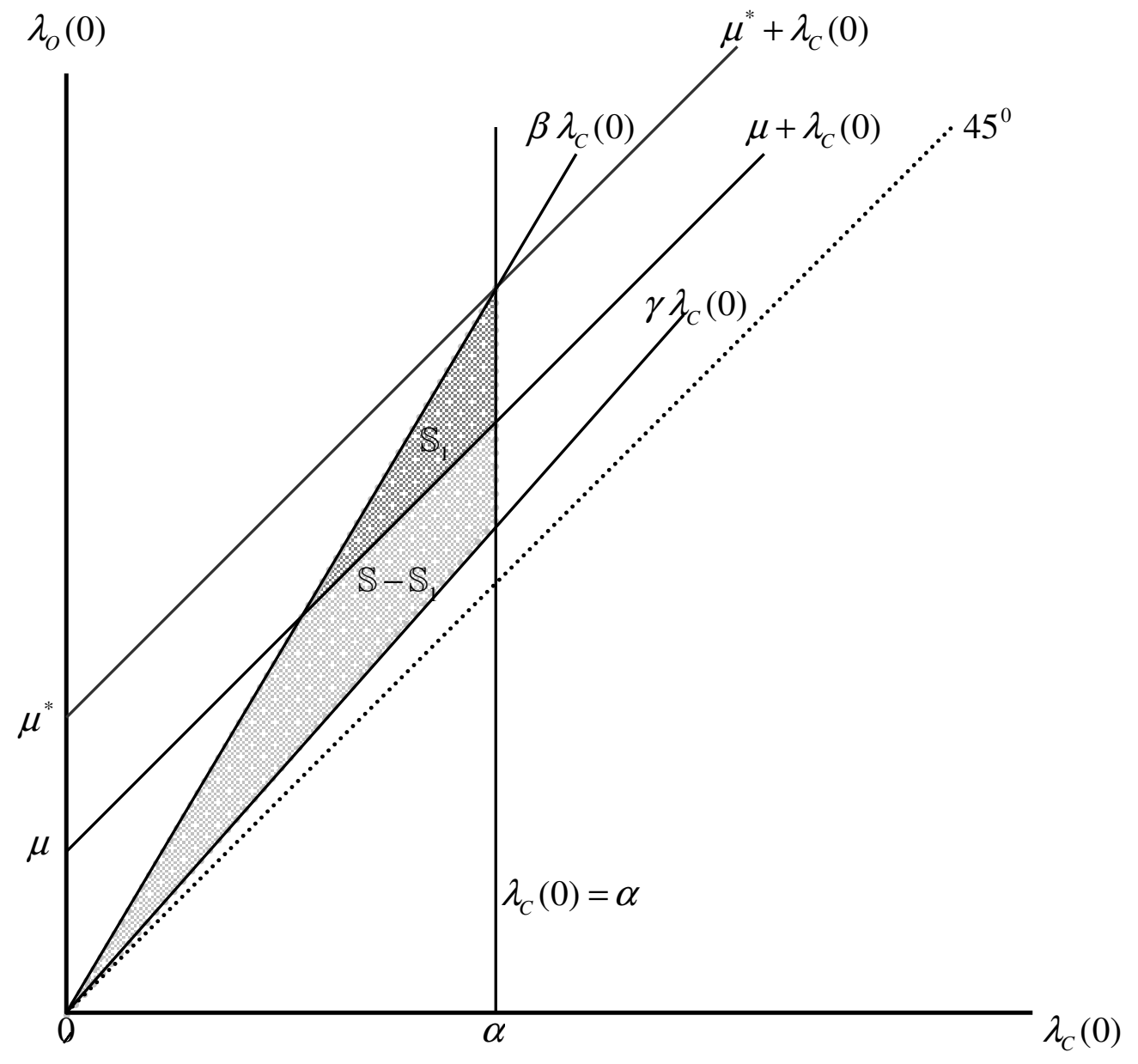

Fig. 2: Coal Extraction is Discontinuous on the Shaded Open Set

The Proposition is symmetric with respect to $E$ and $T$. Fig. 1 shows that if $E$ and $T$ were interchanged, coal would still be extracted discontinuously with $S 1$ and $S 2$ redefined as $0<t_{1 T}<t_{2 T}<t_{1 E}<t_{2 E}<\infty$ and $t_{1 T} \leq 0<t_{2 T}<t_{1 E}<t_{2 E}<\infty$, respectively. 


\section{Conclusion}

This paper provides conditions under which optimal extraction of a nonrenewable resource is discontinuous. At least three resources and two demands are necessary for discontinuous extraction to occur. With many resources and demands, extraction patterns that appear chaotic may be consistent with efficient resource use. 


\section{References}

Amigues, J. P., P. Favard, G. Gaudet and M. Moreaux, 1998, “Optimal Order of Natural Resource Use When the Capacity of the Inexhaustible Substitute is limited," Journal of Economic Theory 80, 153-170.

Chakravorty, U. and D. L. Krulce, 1994, "Heterogeneous Demand and Order of Resource Extraction," Econometrica 62, 1445-1452.

Chakravorty, U., D.L. Krulce, and J. Roumasset, 2005, "Specialization and Nonrenewable Resources: Ricardo meets Ricardo," Journal of Economic Dynamics and Control (in press).

Gaudet, G., Moreaux, M., Salant, S., 2001, "Intertemporal Depletion of Resource Sites by Spatially Distributed Users," American Economic Review 91 (4), 1149-59.

Herfindahl, O.C., 1967, "Depletion and Economic Theory," in M. Gaffney, ed., Extractive Resources and Taxation, (University of Wisconsin Press), 63-90.

M. C. Kemp and N. V. Long, On two folk theorems concerning the extraction of exhaustible resources, Econometrica 48 (1980), 663_673.

Lewis, T. R., 1982, "Sufficient Conditions for Extracting Least Cost Resource First," Econometrica 50, 1081-1083.

Solow R., Wan, F.Y., 1976, "Extraction Costs in the Theory of Exhaustible Resources," The Bell Journal of Economics 7, 359-370. 